\section{Estudos Morfológicos e Funcionais da Microcirculação da Pele no Diabetes Mellitus}

\section{RESUMO}

Os autores analisam os principais métodos de investigação morfofuncional da microcirculação da pele em diabéticos, tanto na literatura médica recente quanto na própria experiência em videocapilaroscopia subungueal, no âmbito dos atuais avanços do conhecimento sobre a fisiopatologia da microangiopatia diabética. Na videocapilaroscopia basal, feita na região periungueal das mãos, ressaltam a importância e a significativa prevalência das alterações morfológicas como edema, microaneurismas, tortuosidade e dilatação do segmento venoso capilar, tanto em indivíduos diabéticos quanto em parentes de $1^{\circ}$ grau com testes de tolerância à glicose normais. Mostram o valor dos estudos das respostas dinâmicas da microcirculação à isquemia e a estímulos farmacológicos, observadas, sobretudo, por medidas de velocidade e/ou fluxo sangüíneo na videocapilaroscopia e dopplerfluxometria a laser, que, na maioria dos trabalhos, estavam alteradas em fases muito precoces da doença, por vasodilatação inadequada. São descritas as medidas do diâmetro e da área do segmento transverso capilar em videocapilaroscopia que podem ser úteis como parâmetrosrespostas ao teste de isquemia reperfusão. Os autores discutem as bases da fisiopatologia que justificam as alterações observadas na videocapilaroscopia tanto basal como dinâmica. (Arq Bras Endocrinol Metab 2003;47/3:271-279)

Descritores: Diabetes mellitus; Microcirculação; Capilaroscopia

\begin{abstract}
Morfologic and Functional Studies of the Skin Microcirculation in Diabetes Mellitus.

The main methods to explore skin microcirculation of diabetic patients and some recent knowledge about physiopathology of diabetic microangiopathy are discussed by the authors based on medical literature and on their experience using videocapillaroscopy. Basal hands nailfold videocapillaroscopy shows high prevalence of edema, microaneurisms and capillar efferent segment tortuosity and dilatation as among diabetic individuals as in first-degree relatives even with normal glucose tolerance tests. Dynamic responses to ischemia and other stimulus suggest precocious inadequate vasodilatatory responses, through flow velocity determinations observed in videocapillaroscopy and laser Doppler flowmetry. Measures of capillary transverse segments area and diameter, in dynamic videocapillaroscopy, are incorporated as ischemia/reperfusion response parameters by the author's experience. Physiopathological aspects of those findings are discussed. (Arq Bras Endocrinol Metab 2003;47/3:271-279)
\end{abstract}

Keywords: Diabetes mellitus; Microcirculation; Capillaroscopy

\section{artigo original}

\author{
Vera L.R.C. Halfoun \\ Therezinha J. Fernandes \\ Maria L.E. Pires \\ Eduardo Braun \\ Magnus G.T. Cardozo \\ Guilherme C. Babbout
}

Departamento de Clínica Médica da Faculdade de Medicina; e Serviços de Angiologia e de

Nutrologia do Hospital Universitário Clementino Fraga Filho - Universidade Federal do Rio de Janeiro, RJ.
Recebido em 08/10/02

Revisado em 07/02/03 Aceito em 04/04/03 
A VISUALIZAÇÃO DIRETA DOS CAPILARES cutâneos, através do uso de lupa estereoscópica, vem sendo obtida na prática, desde o início do século passado, permitindo a análise e diagnóstico de uma série de doenças que afetam a microcirculação (MC). Em nosso meio, Berardinelli, através deste método denominado capilaroscopia, primeiro descreveu o aspecto dos capilares periungueais das mãos de pacientes de sua clínica, inclusive de diabéticos (1). A capilaroscopia na pele permite o estudo de capilares tanto proximais quanto distais na região periungueal de mãos e pés. Tendo em vista que os capilares distais têm função exclusivamente nutridora, seus mecanismos locais de regulação fisiológica não são voltados para regulação térmica da pele. Desta forma, o exame permite o estudo segmentar da MC.

Com o desenvolvimento de lupas mais potentes, foi possível melhorar a sensibilidade óptica e a magnitude das imagens observadas na capilaroscopia. Câmeras de filmagem passaram a ser acopladas a gravadores de videocassete e televisão, com o armazenamento de imagens, método chamado de videocapilaroscopia - VC (2). A VC representou um grande avanço por permitir que os exames gravados pudessem ser analisados repetidas vezes, e, desta forma, a análise de concordância inter e entre observadores melhorou a fidedignidade e confiabilidade dos dados encontrados.

A variação da intensidade de cor, em um determinado ponto da membrana capilar ou do interstício, em intervalos de tempo pré-definidos - densitometria permitiu o estudo da dinâmica da fluoresceína sódica injetada endovenosamente, com objetivo de avaliar a permeabilidade de membrana basal além da morfologia capilar (3).

Mais recentemente, foram desenvolvidos programas de computadores com capacidade de realizar medidas, tanto de dados morfológicos quanto funcionais na MC, como comprimento, área e densidade de vasos; velocidade circulatória; fluxo sangüíneo (4). A determinação destas variáveis quantitativas em computadores acoplados à câmara de filmagem ou gravadores de vídeo, se denominou videofotometria computadorizada - VFC. A VFC possibilitou o estudo dinâmico do comportamento da MC na pele, após estímulos fisiológicos ou patológicos (calor, frio, gravidade, isquemia induzida).

A obtenção de imagens capturadas em computador, em diversos momentos, possibilitou o estudo dinâmico do comportamento da MC na pele, após estímulos fisiológicos ou patológicos (calor, frio, gravidade, isquemia induzida) ou farmacológicos (injeções de contrastes específicos, drogas, e outros), processo denominado videocapilaroscopia dinâmica - VD (figura 1). Este método inovou também pela forma de gravação dos exames em CD (discos compactos), melhorando a qualidade das imagens e condições de armazenamento (5-7).

A visualização dos capilares, arteríolas, vênulas e pequenas artérias e veias da pele e também de mucosas tem sido feita atualmente com equipamento denominado Cytoscan E-II (Cytometrics Inc., Phi, USA) com imagem espectral ortogonal polarizada, já estando disponíveis dados de análise destas imagens tanto em repouso quanto após estímulos e injeção de contrastes (8). Não encontramos dados referentes a estes estudos em diabéticos.

Métodos de estudo da MC da pele como a dopplerfluxometria a laser (DPL), oximetria tissular, pletismografia e tensimetria capilar, entre outros, viabilizaram o estudo de variáveis como fluxo sangüíneo/perfusão, oxigenação tissular, débito circulatório e pressões intracapilares, respectivamente. $\mathrm{O}$ estudo simultâneo destes métodos com a VD permite uma avaliação funcional global da MC (2,9-10).

Desta forma, nos últimos dez anos, uma boa e relevante quantidade de informações sobre o comportamento da MC no diabetes vem sendo obtida para a melhor compreensão desta doença no que se refere à gênese de suas complicações. Os estudos bioquímicos, anatomopatológicos e clínicos têm contribuído ainda mais para a evolução destes conhecimentos.

Os objetivos deste trabalho são a revisão e atualização sobre métodos de estudo da $\mathrm{MC}$ da pele no diabetes mellitus, à luz dos recentes avanços do conhecimento sobre a fisiopatologia da microangiopatia diabética e da experiência dos autores.

\section{Anatomia e Fisiologia da Microcirculação}

A MC pode ser entendida como a unidade funcional do sistema circulatório que reúne uma série de estru-

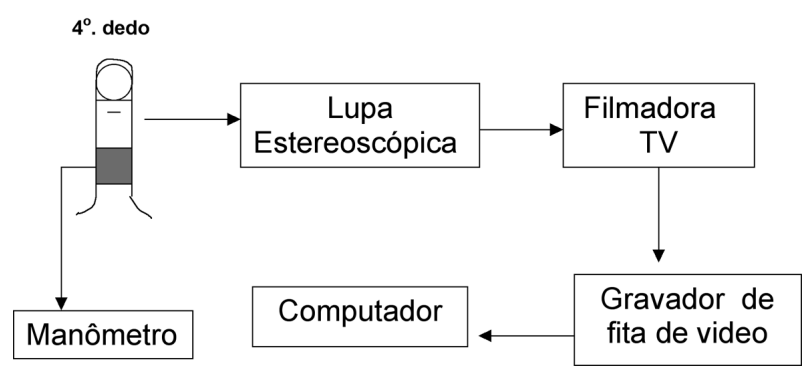

Figura 1. $O$ esquema representa $\mathrm{o}$ equipamento utilizado com armazenamento em fitas cassette. No entanto, a câmara de filmagem pode estar acoplada diretamente ao computador, neste caso armazenando-se as imagens em disco compacto através de gravador de CD. 
turas com funções particulares e harmônicas, composta de pequenas artérias, arteríolas, metarteríolas, capilares, pré-vênulas, vênulas, pequenas veias, comunicações arterio-venosas e arteríolo-venulares, linfáticos e estruturas perivasais, imersas em uma substância amorfa chamada de substância fundamental (figura 2). Todos os elementos desse conjunto apresentam funções específicas que permitem ajustes circulatórios necessários ao funcionamento adequado de cada tecido ou de cada órgão. Deste modo, a MC está intimamente adaptada às exigências metabólicas dos tecidos, sendo esta adaptação de caráter anatômico e funcional.

A MC da pele apresenta duas grandes funções hemodinâmicas: (1) manutenção do débito sangüíneo "nutritivo" da pele (ação loco-regional), que participa das trocas metabólicas entre o sangue e os tecidos cutâneos; e (2) manutenção do débito sangüíneo "anastomótico" que se faz pelas ligações diretas entre pequenas artérias e pequenas veias e, também, entre arteríolas e vênulas, sem função nutritiva, fundamental para a termorregulação corporal (ação sistêmica) (11).

$\mathrm{Na}$ pele, a termorregulação sistêmica é responsável por $75 \%$ do débito sangüíneo total, enquanto o restante do fluxo local é dirigido ao débito metabólico. Esta característica fisiológica explica situações aparentemente paradoxais, tais como a existência de isquemia residual na presença de fluxo sangüíneo global preservado, caracterizando o débito anastomótico predominando sobre o nutricional, tal como ocorre na pele dos pacientes diabéticos (11).
A avaliação da resposta vascular à isquemia induzida em diabéticos, na videocapilaroscopia, é feita através da observação dos capilares nutridores da pele, enquanto a dopplerfluxometria a laser analisa todo o conjunto de funções nutridora e termorreguladora.

A regulação do fluxo na microcirculação depende da plasticidade do capilar e dos elementos figurados do sangue, principalmente hemácias, e da viscosidade plasmática, que são os determinantes da viscosidade total do sangue que, quando normal, permite um fluxo laminar até o nível de pequenas artérias. A plasticidade das hemácias é muito importante, possibilitando que ela se achate para passar ao longo do capilar, cujo diâmetro é menor. A viscosidade plasmática depende do teor de proteínas de alto peso molecular (albumina, fibrinogênio, alfa2 globulinas e outras) e do grau de hidratação. Nos capilares nutridores, além do tonus basal da musculatura das metarteríolas, o fluxo depende da abertura e fechamento do esfíncter pré-capilar, que é instável, intermitente e às vezes retrógrado, caracterizando um fluxo perturbado (12).

A regulação da motricidade dos vasos na microcirculação se faz por mecanismos centrais, regionais e locais. As ações centrais são reguladas pelos baroceptores carotidianos, pelos sistemas renina-angiotensina e calicreína-quininas e ainda pela regulação hormonal e renal da quantidade de sódio e água, mecanismos que regulam o débito para a microcirculação. A ação regional neural se faz sobre pequenas artérias, arteríolas, metarteríolas, em sua maioria com receptores alfa

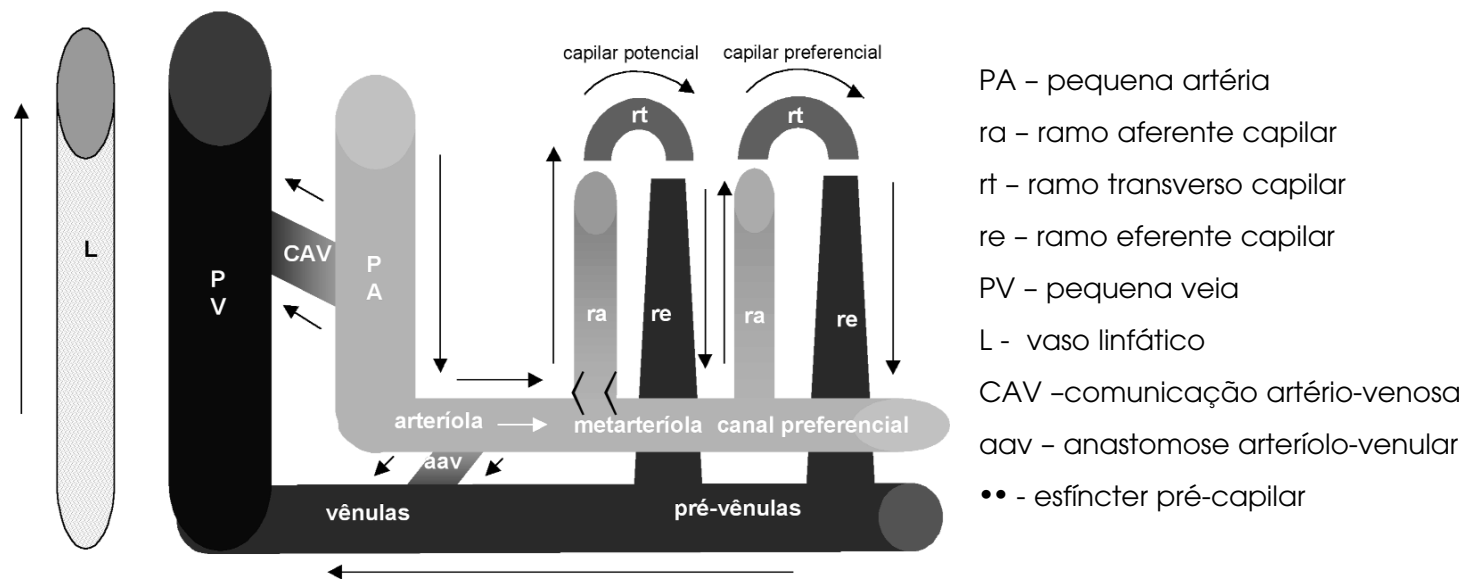

Figura 2. A MC cutânea, estudada como uma unidade funcional hipotética, apresenta os seguintes componentes: A) pequenas artérias terminais onde se encontram as comunicações artério-venosas - F - que derivam o sangue diretamente para as pequenas veias e apresentam camada muscular bem desenvolvida, com ampla inervação simpática e colinérgica, sendo esta última responsável pela ação vasodilatadora, importante na função reguladora da pressão arterial e na termorregulação. Estas artérias se ramificam progressivamente dando origem às arteríolas e metarteríolas, que, por sua vez, originam os capilares ou canais preferenciais (por onde passam 75\% do fluxo sangüíneo) ou os capilares potenciais (responsáveis pelas trocas metabólicas. Os capilares são formados por ramos aferente (B), transverso (C) e eferente (D). As arteríolas podem gerar anastomoses arteríolo-venulares que se abrem por ação metabólica local. Os capilares vão desaguar no sistema venoso (pré-vênulas, vênulas e pequenas veias) com enervaçăo adrenérgica. Os linfáticos fazem parte da unidade microcirculatória e sua função é importante na retirada de macromoléculas do interstício. 
adrenérgicos e ação vasoconstrictora; sobre comunicações arterio-venosas, com fibras simpáticas colinérgicas, e, portanto, vasodilatadoras; e, finalmente, sobre vênulas e pequenas veias, ambas com receptores adrenérgicos, respondendo com vasoconstricção (12). A regulação local se faz pelo endotélio através da liberação de várias substâncias por ele produzidas. Em condições normais, o mais importante fator de relaxamento arterial é mediado pelo óxido nítrico derivado do endotélio (e-NO), sintetizado em resposta a diversos estímulos, principalmente a hipóxia. Pode-se, inclusive, estudar a resposta vasodilatadora de um vaso pela indução de hipóxia e/ou injeção de acetilcolina. A hiperemia fisiológica reflete a integridade na produção de e-NO, enquanto a vasodilatação produzida pela injeção de nitroglicerina ou derivados (doadora de $\mathrm{NO}$ ), reflete integridade estrutural do vaso (resposta independente de e-NO). Por outro lado o mais importante constrictor é a endotelina l (13). No diabetes ocorre disfunção endotelial conforme será discutido mais adiante.

\section{Estudos da Microcirculação da Pele no Diabetes}

Os principais estudos descritivos dos capilares na região periungueal de diabéticos adultos, em repouso, feitos através da capilaroscopia e/ou VC, por vários autores, demonstraram as seguintes alterações morfológicas:
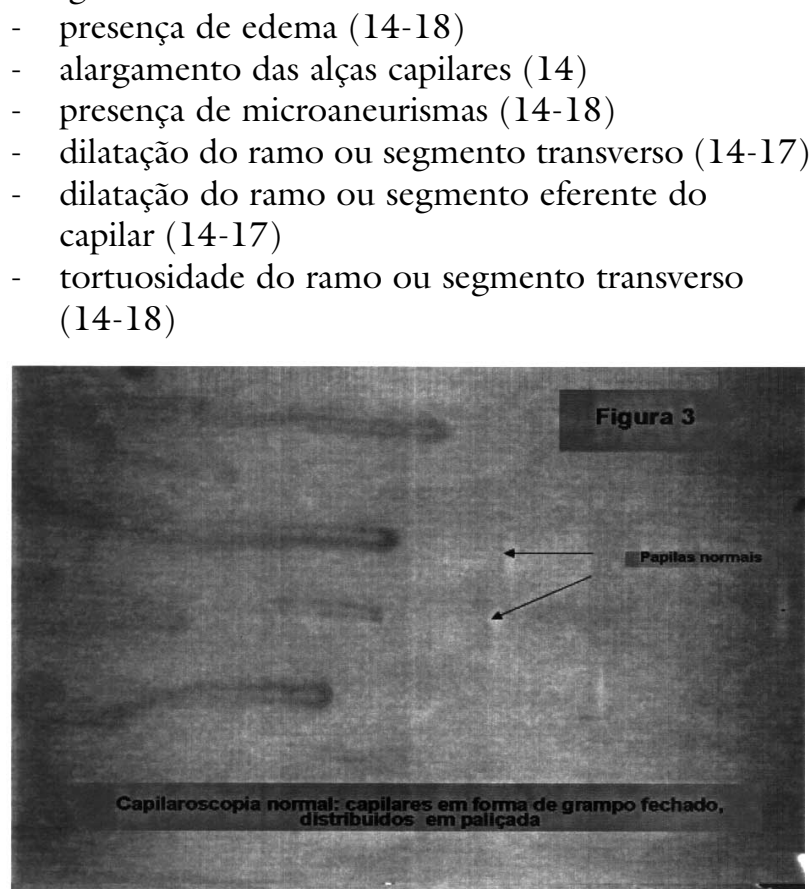

Figura 3. Capilaroscopia normal: capilares em forma de grampo fechado, distribuídos em paliçada. tortuosidade do ramo ou segmento eferente (14-18)

- ramificações do capilar (19)

- hemorragias $(19,20)$

Há consenso, nos achados, de freqüência aumentada de edema, microaneurismas, dilatação de segmento transverso e tortuosidade do ramo eferente, em diabéticos em relação a indivíduos normais, pela maioria dos autores (figuras 3 a 6) (14-20). O edema tem sido reconhecido pela presença de retificação de papila dérmica, visibilidade difícil e presença de névoa translúcida que apaga parcialmente o capilar (figuras 4 a 6), podendo levar a um aspecto de encurtamento - a imagem de "ponto e vírgula" ou de "banca de peixe" (figura 6).

Em nossa experiência, em amostra aleatória de 37 casos de diabetes tipo 2, edema, microaneurisma, dilatação de segmento transverso e tortuosidade do ramo eferente estiveram presentes em 62, 24, 25 e $45 \%$, respectivamente $(\mathrm{p}<0,05$ em relação a não diabéticos para todas as variáveis).

Fahrig e cols. (19) encontraram, na conjuntiva de grupo de 100 pacientes diabéticos do tipo 2, ramificação de vasos em $45 \%$, independente da duração da doença, da existência ou não de neuropatia e do tipo de tratamento. Diagnosticaram, em 20\%, hemorragias, também observadas por Fenton e cols., em grupo semelhante de pacientes. Estes achados, no entanto, não foram confirmados pela maioria dos autores (19-20).

Bernardini (21), em 72 parentes de $1^{\circ}$ grau de diabéticos, encontrou prevalências de $41,9 \%$ de edema, $36,8 \%$ de microaneurismas, $29,7 \%$ de tortuosidade do ramo eferente e $35,7 \%$ de encurtamento de alças, estatísticamente significativas em relação à encontrada em controles $(\mathrm{p}<0,05)$. Uma amostra de 17 parentes de $1^{\circ}$ grau de diabéticos estudada por nós mostrou freqüências de $60,28,36$ e $32 \%$ destas variá-

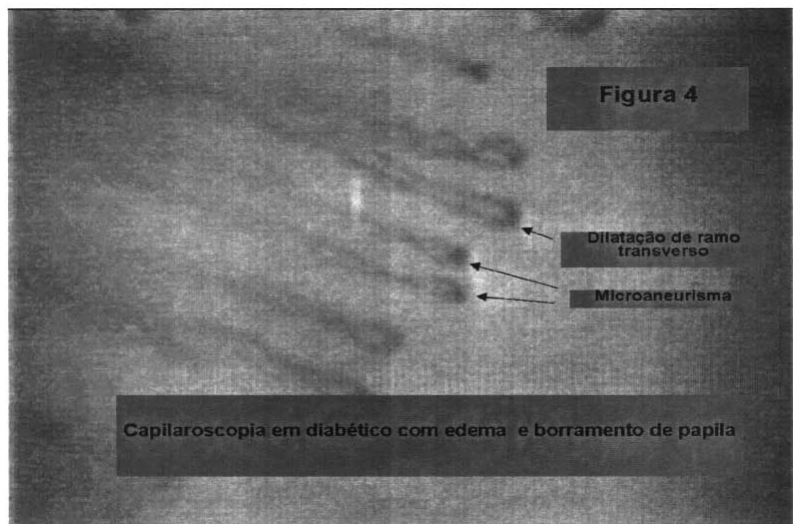

Figura 4. Capilaroscopia em diabético com edema e borramento de papila.

Arq Bras Endocrinol Metab vol $47 n^{\circ} 3$ Junho 2003 


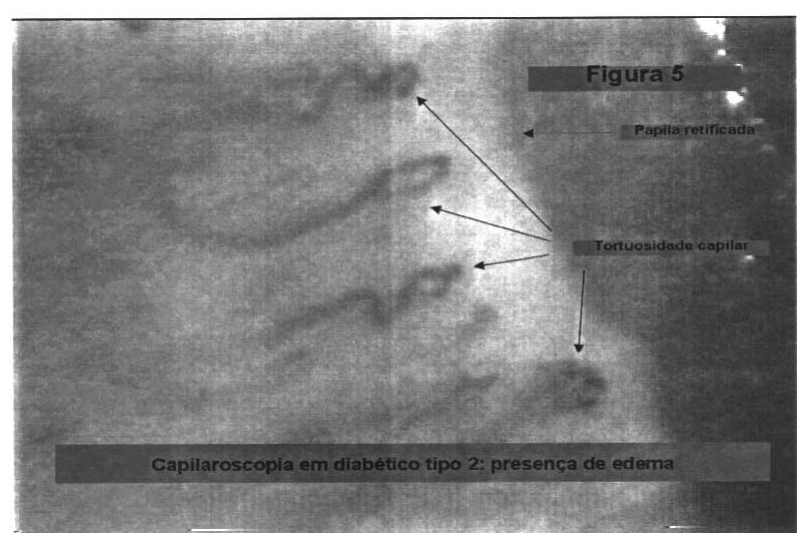

Figura 5. Capilaroscopia em diabético tipo 2: presença de edema

veis, todas significativamente mais freqüentes do que em não diabéticos, sem história familiar da doença. $\mathrm{A}$ redução da densidade capilar foi encontrada em indivíduos não diabéticos com resistência à insulina por Irving e cols. Este autor encontrou correlação negativa entre glicemia de jejum e densidade capilar (22).

Trabalho realizado com 18 controles, 22 parentes de $1^{\circ}$ grau e 37 diabéticos tipo 2 mostrou que a medida da largura dos capilares varia, de 15 a $55 \mu$ (média $=32,22 \pm 8,26 \times 35,25 \pm 7,25 \mu$ ) nos grupos de diabéticos tipo 2 e parentes, respectivamente, quando comparados aos controles (média $=32,65 \pm 13,42 \mu$ ), diferenças não significativas estatisticamente $(p=0,66)$. $\mathrm{O}$ diâmetro apical médio nos controles, parentes e diabéticos foi de $13,42 \pm 3,15$ X $14,99 \pm 3,28$ X $14,44 \pm 3,95 \mu$, também semelhantes $(\mathrm{p}=0,35)$, da mesma forma que a área do segmento transverso

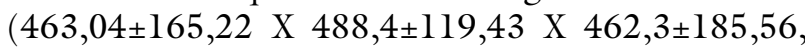
$\mathrm{p}=0,82)$. Entre os controles não houve correlação significativa entre as medidas de diâmetro apical e as idades dos pacientes, bem como com índices de massa corporal $(\mathrm{p}=0,21 \mathrm{e} \mathrm{p}=0,77)$, porém houve correlação positiva $(\mathrm{r}=0,7 \mathrm{l}$ e $\mathrm{p}=0,0008) \mathrm{com}$ as áreas do segmento transverso (23).

Os capilares parecem ser maiores em homens do que em mulheres não diabéticos, quando avaliados pela medida do diâmetro apical e/ou área do segmento transverso $(14,41 \mathrm{X} \mathrm{12,62 \mu ,} \mathrm{p}=0,20$ e $543,89 \mathrm{X}$ $396,42 \mu^{2}$, em homens e mulheres respectivamente), porém esta diferença não foi estatisticamente significativa na amostra estudada $(\mathrm{p}=0,06)(23)$.

$O$ teste de isquemia/reperfusão (TIR) vem sendo feito pela VD, usando-se esfigmomanômetro insuflado acima da pressão arterial máxima, durante $\mathrm{l}$ a 3 min na região bicipital ou na base do $4^{\circ}$ dedo da mão dominante. A velocidade circulatória, registrada por programas de computador como CAP Show ou pela

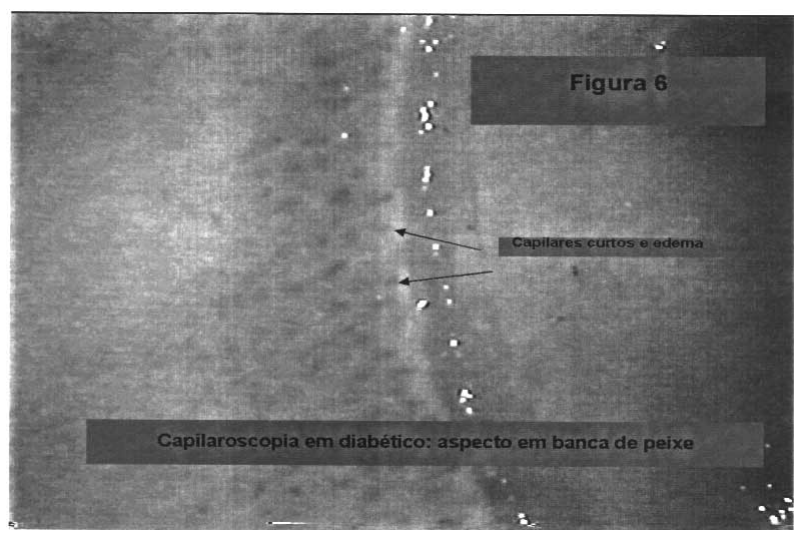

Figura 6. Capilaroscopia em diabético: aspecto em banca de peixe

técnica de "dupla janela", em imagens capturadas durante curtos intervalos após a descompressão, se eleva significativamente menos nos diabéticos em relação a controles saudáveis, e o tempo para obtenção do incremento máximo é maior $(5,7)$. Respostas análogas são vistas quando se estimula vasodilatação pelo calor ou exercício com menor incremento da velocidade circulatória em diabéticos do que não diabéticos.

A medida da expansão da área do segmento transverso capilar projetada na televisão e capturada em computador durante a reperfusão pode ser um bom parâmetro de avaliação do teste de isque$\mathrm{mia} /$ reperfusão em VD (figura 7) (24). Em uma amostra não selecionada de 34 diabéticos tipo 2 , estudados por nós através deste método, a expansão máxima foi semelhante aos não diabéticos, porém houve um aumento estatisticamente significativo do tempo para alcançar o incremento máximo de área. Em 10 diabéticos tipo 2 recém diagnosticados descompensados e sem complicações, ocorreu, também, além do aumento do tempo, aumento significativo de incremento de área máxima durante a reperfusão, quando comparados a diabéticos compensados (25).

Os trabalhos sobre isquemia induzida, utilizando-se a medida da perfusão cutânea pela DPL, são semelhantes aos feitos com VD, demonstrando também diminuição e retardo do incremento do fluxo em diabéticos durante a reperfusão $(9,10)$.

Jörneskog e cols. estudaram a resposta à isquemia induzida na microcirculação dos pés pela VCD e DPL em 10 controles e 20 pacientes diabéticos tipo 1 , sendo 10 com e 10 sem complicações microvasculares, todos com boa função troncular. Não houve diferença nos resultados de DPL entre diabéticos e controles, porém na VCD houve significativa redução do pico máximo de velocidade, assim como retardo no tempo para alcançá-la, indicando má dis- 


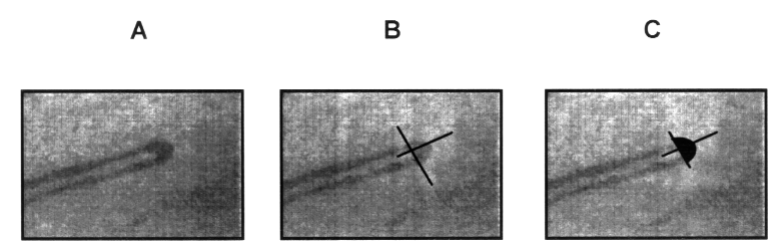

Figura 7: Etapas na definição da área do segmento transverso: A - capilar normal; B - linha tangente ao bordo interno do arco transverso do capilar, perpendicular ao seu maior eixo; C - área do segmento transverso.

tribuição regional do sangue na microcirculação, com alteração precoce dos capilares nutridores (26).

Arora e cols. (27), estudando 15 diabéticos com neuropatia e 14 sem esta complicação, pela DPL após injeção de acetilcolina (estimuladora de vasodilatação endotélio-dependente) e de nitroprussiato de sódio (estimulador de vasodilatação independente de endotélio), não observaram diferenças nos resultados dos pés e mãos de diabéticos, porém houve resposta significativamente alterada nos pés de pacientes neuropatas, após injeção de acetilcolina, mostrando que as disfunção endotelial, em neuropatas é mais precoce e grave em membros inferiores. Por outro lado, o estímulo contralateral da mão pelo frio, em controles, 12 diabéticos com neuropatia e 12 sem esta complicação, estudados por Haak e cols. (28), levou a menor redução da velocidade circulatória no membro contralateral $(0,29 \times 0,42 \mathrm{~mm} / \mathrm{seg}, \mathrm{p}<0,03)$ nos diabéti$\cos$, independente da existência de neuropatia.

Tur e cols. (29), utilizando DPL e VD das mãos, após isquemia induzida, em diabéticos tipo 2 com e sem retinopatia diabética, mostraram redução na relação entre o incremento do pico máximo do fluxo e o tempo de sua obtenção em ambos os grupos, sendo mais significativa e mais intensa no grupo com retinopatia. Ambos os grupos tinham bom controle metabólico e os seus resultados não tiveram relação com índice de massa corporal ou duração da doença.

Meyer e Schatz (30), pela DPL, estudaram a reatividade microvascular em 16 diabéticos tipos 1,19 do tipo 2 e controles com idade e sexo pareados, subdivididos em grupos - com bom (HbAlc $<7,5 \mathrm{~g} \%$ ) e mau (HbAlc $\geq 7,5 \mathrm{~g} \%$ ) controle. No diabetes tipo 1, encontraram redução de incremento máximo de fluxo e aumento do tempo para alcançá-lo em todos os pacientes, independente do controle metabólico ou duração da doença (> ou < 10 anos), porém, no diabetes do tipo 2, estas alterações na reperfusão só ocorreram no grupo com mau controle, independente da duração da doença. Ele conclui que, no tipo 2, o controle metabólico é importante na gênese da disfunção endotelial (30).
Parece, portanto, haver uma relação entre disfunção endotelial $\mathrm{X}$ existência de complicações microvasculares e/ou mau controle metabólico.

Em nossa experiência, em VD, a área máxima do segmento transverso capilar, durante a reperfusão pós isquemia, foi semelhante em controles e diabéticos tipo 2 com frutosaminemia menor do que $3 \mathrm{mg} \%$, sem complicações vasculares e/ou hipertensão arterial, porém o tempo para obtê-la foi significativamente maior, mostrando haver alterações discretas mesmo nos indivíduos compensados recentemente (31).

Em parentes de diabéticos, a resposta vascular pode estar alterada ou não, não havendo consenso na literatura. Caballero e cols., em interessante trabalho, comparou pela ultrassonografia o diâmetro da artéria braquial, e pela DPL, a reatividade vascular pós injeção de acetilcolina para avaliação da reatividade endotélio dependente, em controles, parentes com TOTG normal, TOTG anormal e diabéticos sem complicações. A reatividade microvascular foi reduzida em diabéticos e parentes, havendo correlação inversa com a pressão arterial, glicemia de jejum, HDL colesterol (HDL-c), insulinemia basal e grau de resistência à insulina pelo HOMA (bomeostatic model assessment). A vasodilatação endotélio-dependente foi correlacionada inversamente com o índice de massa corporal e a pressão diastólica. O diâmetro da artéria braquial se correlacionou positivamente com a resistência à insulina, pressão sistólica, hemoglobinemia Alc e HDL-c (26). Estes autores sugeriram deficiência vasodilatatória endotélio-dependente (32). Em nossa experiência, em VD, não houve diferenças na medida da área máxima do segmento transverso do capilar após descompressão do manguito em 34 indivíduos parentes de $1^{\circ}$ grau de diabéticos do tipo 2, tanto nos com TOTG normal quanto naqueles com intolerância à glicose (33).

Estudos de perfusão sangüínea podem ser feitos pela pletismografia, através do registro da volumetria sangüínea nas extremidades dos membros $(34,35)$. Eles também confirmam que ocorre redução do seu incremento máximo pós-isquemia em diabéticos. A oximetria da pele, em alguns trabalhos, é concordante com a redução da reatividade microvascular, pois demonstra redução de transporte de oxigênio tissular em diabéticos, sugerindo hipóxia na microcirculação (35). Sandeman e cols. (36), medindo a pressão intracapilar no ramo transverso capilar pela canulação direta do vaso, demonstraram com elegância o aumento médio de pressão capilar, existente em 21 diabéticos do tipo 1 , em relação a 29 controles saudáveis, pareados por sexo e idade, contribuindo para diminuir o incremento da velocidade circulatória. Esta alteração pode ser decor- 
rente de redução do gradiente de pressão arteríolovenular, por aumento de pressão venular média, tal como ocorre na insuficiência venosa crônica, justificando a dilatação e tortuosidade dos segmentos eferentes, vistos em VC. Em 7 pacientes, eles estudaram a pressão capilar antes e depois da obtenção do controle metabólico, havendo queda significativa da pressão após o controle da doença $(\mathrm{p}=0,02)$.

\section{Alguns Aspectos da Fisiopatologia da Microcirculação no Diabetes}

A presença destas alterações morfológicas e funcionais na microvasculatura de diabéticos vem sendo atribuída a vários distúrbios fisiopatológicos existentes nesta doença, que se relacionam entre si: (i) disfunção endotelial, (ii) viscosidade sangüínea e plasmática aumentadas, (iii) tendência à trombogênese e diminuição da fibrinólise e, (iv) aumento da permeabilidade da membrana basal.

Estas alterações podem se relacionar com alguns mecanismos metabólicos básicos na fisiopatologia do diabetes mellitus: resistência à insulina, estresse oxidativo, glicosilação não enzimática e ativação das via do poliol (figura 8) (37-40).

A disfunção endotelial, tanto em artérias quanto na microcirculação, está bem associada à resistência à insulina e ao estresse oxidativo, conforme demonstrado por vários autores (37-40). Este fato ocorre por múltiplas causas, desde a ação direta da hiperglicemia e hiperinsulinemia, capazes de aumentar a liberação de endotelina $\mathrm{l}$ (principal vasoconstrictor gerado no endotélio), assim como a ação da hiperglicemia na redução da biodisponibilidade de e- $\mathrm{NO}$ (vasodilatador endotélio-derivado mais potente), gerando uma capacidade vasodilatadora defeituosa (13,37-40). Esta alteração, provavelmente, explica a redução do incremento de velocidade circulatória e da perfusão pósisquemia em diabéticos, observada na VD e na DPL.

A disfunção endotelial, além de acarretar dismotilidade no capilar e artérias, pode se expressar por aumento de fatores antifibrinolíticos (PAI-l - fator inibidor de ativação do plasminogênio) e por alterações características da inflamação com aumento de permeabilidade capilar (ativação de citocinas e de moléculas de adesão à membrana) e conseqüente edema, visualizado na VC (figura 8).

O aumento do inibidor de ativação do plasminogênio 1 (PAI-1), entre outros, secretado principalmente no endotélio do tecido adiposo, encontrado nos casos de resistência à insulina, está associado à inibição da fibrinolise. A diminuição de prostaciclina PGI 2, causada por alterações do metabolismo do ácido aracdônico no diabetes, acarreta redução de vasodilatação arterial e aumento da agregação plaquetária. Estas disfunções estão também aliadas à tendência à trombogênese por outros fatores, de causa menos esclarecida, como o aumento do fator de von Willebrand, dos fatores VIIc e VIII, do fibrinogênio sérico, e à disfunção plaquetária (hiperagregação espontânea $\mathrm{e}$ após estímulo por ADP) observados no diabetes. A tendência à trombose e a atividade antifibrinolítica encontradas no plasma de diabéticos podem justificar a redução da densidade capilar, encontrada por alguns autores na VD, por microtrombose (12).

A glicosilação não enzimática de proteínas, como enzimas, e de estruturas constituintes da membrana basal, e a redução na ATPase $\mathrm{Na}-\mathrm{K}$ conseqüente à ativação da vias do poliol, que ocorrem no diabetes, podem explicar disfunções em processos metabólicos intra e extracelulares e alterações de transporte de mem-

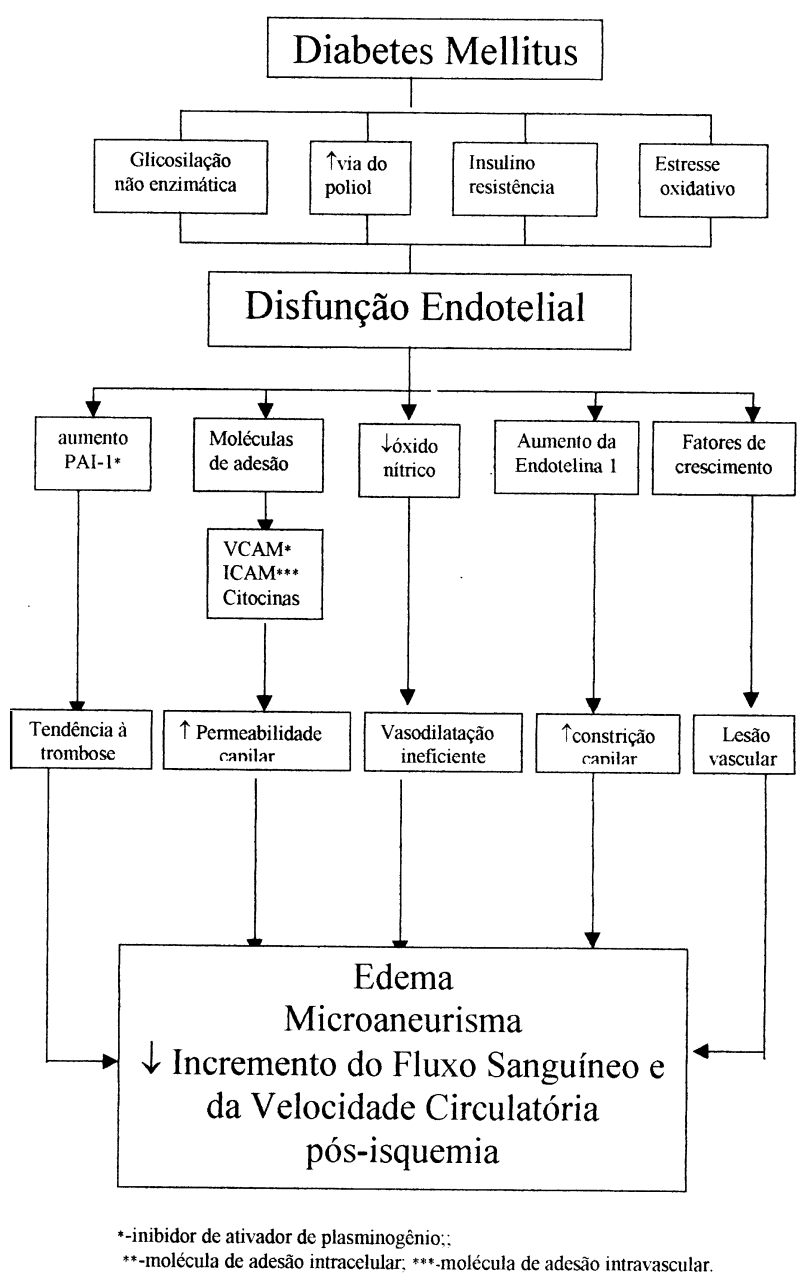

Figura 8: Hipóteses sobre a fisiopatologia da microcirculação no diabetes mellitus 
branas, existentes na doença, incluindo a disfunção endotelial. O aumento dos níveis de hemoglobina Alc, por exemplo, justifica, em parte, a hipóxia na microcirculação e os distúrbios de fluxo encontrados no capilar, observados em VD. A hipóxia é agravada pela vasodilatação compensatória insuficiente, edema e presença de microtrombos, perpetuando-se o ciclo. Ela tem sido responsabilizada pela degeneração do pericito com aparecimento de microaneurismas (12,37-40).

\section{CONCLUSÔES}

A VC, VD e VFC são métodos simples, não invasivos, ainda não incorporados na prática clínica, no que se refere ao diagnóstico do diabetes mellitus e suas complicações. No entanto, são capazes de demonstrar, precocemente, alterações morfo-funcionais que podem preceder o aparecimento da microangiopatia diabética. Em descendentes de diabéticos, sua importância como marcador da doença precisa ser avaliada, principalmente naqueles com resistência à insulina.

As alterações encontradas decorrem de má regulação do fluxo local por disfunção endotelial, acarretando respostas vasodilatatórias inadequadas, causadas, principalmente, pela redução de biodispnibilidade de e-NO e diminuição de endotelina 1 , aliada a outras alterações funcionais da MC.

Novas pesquisas de longo prazo necessitam ser feitas para o estabelecimento do valor prognóstico das alterações encontradas nos métodos de exploração da $\mathrm{MC}$, tanto no que se refere ao aparecimento do diabetes, quanto de suas complicações vasculares.

\section{AGRADECIMENTOS}

Os autores agradecem ao Laboratório Servier pelo auxílio na compra do equipamento utilizado na realização de alguns trabalhos feitos pela equipe, citados neste artigo.

\section{REFERÊNCIAS}

1. Berardinelli W. Semiologia parvivascular. Tese, Rio de Janeiro. Oficinas Gráficas Alba, 1939,LXI.

2. Fagrell B, Fronek A, Intaglietta M. A microscope television system for studying flow velocity in human skin capillaries. Am J Physiol 1977:233:H318-21.

3. Bollinger A, Frey J, Jäger K, Furrer J, Seglias J, Siegenthaler W. Patterns of diffusion through skin capillaries in patients with long term diabetes. N Eng J Med 1982;307:1305-10
4. Fagrell B, Rosen L, Ericksson SE. Comparison between a new computerized and an analogue video-photometric, crosscorrelation technique for measuring capillary blood cell velocity in humans. Int J Microcir Clin Exp 1994; 14:133-4.

5. Fagrell B, Hermansson IL, Karlander SG. Vital capillary microscopy for assessment of skin viability and microangiopathy in patients with diabetes mellitus. Acta Med Scand $1984 ; 687: 25-8$

6. Gasser P, Berger W. Nailfold videomicroscopy and local cold test in type 1 diabetics. Angiology 1992;43:395-400.

7. Pangratis N. Diagnostic investigation using vital capillary microscopy and dynamic capillaroscopy. Clin Hemorheol Microc 1997; 17:371-83.

8. Harris AG, Sinitsina I, Messmer K. The Cytoscan model E II, a new reflectance microscope for intravital microscopy: comparison with standard fluorescence method. J Vasc Res 2000;37:469-76.

9. Meyer MF, Schatz H. Assessment of diabetics alterations of microcirculation by means of capillaroscopy and laser-doppler anemometry. Med Klin 2001;15:96 (abstract).

10. Carpentier PH. New techniques for clinical assessment of the peripheral microcirculation. Drugs 1999;58(spec 1):17-22.

11. Mello NA. Noções de Microcirculação. In: Mello NA, editor. Angiologia. Rio de Janeiro: Guanabara-Koogan, 1998.p.29-41.

12. Boisseau MR. Régulation vasomotrice de la microcirculation. In: Vayssairat M, Carpentier P, eds. Microcirculation clinique. Paris: Masson, 1996.p.40-70.

13. Wajchenberg BL. Disfunção endotelial no diabetes do tipo 2. Arq Bras Endocrinol Metab 2002;46:514-9.

14. Pazos Moura CC, Moura EG, Bouskela E, Torres Filho IP, Breittenbach MM. Nailfold capillaroscopy in diabetes mellitus: Morphological abnormalities. J Vasc Biol 1990;2:52-5.

15. Mouthon JM, Grimaldi A, Wechsler B, Thervet F, Goudeau P. Diabetic microangiopathy. Role of capillaroscopy. Presse Med 1989; 18:1647-50.

16. Josa G, Salvi P, Zoli I, Battistini G, Pretolani E. Capillaroscopy and diabetic microangiopathies. J Mal Vasc $1989 ; 14: 55-6$

17. Morais IC, Halfoun VLRC, Fonseca MHG, Oliveira JEP, Bernardini EMT. Capilaroscopia em diabéticos. Arq Bras Endocrinol Metab 1988;32:18-23.

18. Fernandes TJ, Bernardini EMT, Halfoun VLRC. Capilaroscopia em crianças diabéticas. Arq Bras Endocrinol Metab 2001;45:441-6.

19. Fahrig C, Breitinger L, Heidrich $\mathrm{H}$. Vital capillary microscopic findings in the nailfold of patients with diabetes mellitus. Vasa 2000;29:258-3.

20. Fenton BM, Zweifach BW, Worthen DM. Quantitative morphometry of conjunctival microcirculation in diabetes mellitus. Microvasc Res 1979; 18:153-66.

21. Bernardini E. Estudo da microcirculação em descendentes de diabéticos através da capilaroscopia. Tese de doutorado em Cirurgia Vascular. Escola Paulista de Medicina, São Paulo. 1992. 
22. Irving RJ, Walker BR, Noon JP, Watt GC, Webb DJ, Shore AC. Microvascular correlates of blood pressure, plasma glucose, and insulin resistance in health. Cardiovasc Res 2002;53:271-6.

23. Pires MLE, Bahbout GC, Braun E, Halfoun VRLC, Fernandes TJ. Avaliação da área basal do segmento transverso capilar (ABSTC) pela videocapilaroscopia em indivíduos saudáveis, diabéticos do tipo 2 e em parentes de $1^{\circ}$ grau. Arq Bras Endocrinol Metab 2002;46(3, supl1):S256 (resumo).

24. Halfoun VLRC, Fernandes TJ, Pires MLE, Victer F, Tavares $\mathrm{R}$, Cardozo $\mathrm{M}$, et al. Videocapilaroscopia e diabetes mellitus: projeção da área do segmento capilar transverso em processamento de imagem. Arq Bras Endocrinol Metab 2001;45(5, supl1):S584 (resumo).

25. Fernandes TJ, Halfoun VLRC, Pires MLE, Oliveira JEP, Cardozo M. Videocapillaroscopy in diabetes type 2: effects of metabolic control on microvascular response to induced ischaemia. Diabetologia 2002;45(suppl 2):A383.

26. Jörneswog G, Brisman K, Fagrell B. Skin capillary circulation is more impaired in the toes of diabetic than nondiabetic patients with peripheral vascular disease. Diabetic Med 1995; 12:36-41.

27. Arora S, Smakowsky P, Freyberg RG, LoGerfo F, Veves A. Differences in foot and forearm skin microcirculation in diabetic patients with and without neuropathy. Diabetes Care 1998;21:1339-44.

28. Haak E, Haak E, Grözinger Y, Krebs G, Usadel KH. The impact of contralateral cooling on skin capillary blood cell velocity in patients with diabetes mellitus. J Vasc Res 1998;35:245-9.

29. Tur E, Yosipovitch $G$, Bar-On Y. Skin reactive hyperemia in diabetic patients. A study by laser doppler flownmetry. Diabetes Care 1991;14:958-61.

30. Meyer MF, Schatz H. Influence of metabolic control and duration of disease on microvascular dysfunction in Diabetes assessed by laser Doppler anemometry. Exp Clin Endocrinol. Diabetes 1998; 106:395-403.

31. Fernandes TJ, Pires MLE, Halfoun VLRC, Cardozo M Videocapilaroscopia dinâmica em diabéticos do tipo 2: efeitos do controle metabólico sobre a resposta vascular à isquemia induzida. Arq Bras Endocrinol Metab 2002;45(5, supl1):S257 (resumo).
32. Caballero $A E$, Arora Ssaonaf $R$, Lim SC, Smakowski $P$, Parti JW, King GL, et al. Microvascular and macrovascular reactivity is reduced in subjects at risk for type 2 diabetes. Diabetes 1999;48:1856-62.

33. Pires MLE, Halfoun VLRC, Fernandes TJ, Cardose M. Videocapilaroscopia em diabéticos do tipo 2 e em parentes de $1^{\circ}$ grau: resposta vascular à isquemia induzida. Arq Bras Endocrinol Metabol 2002;46(3, supl1):S256.

34. Vayssairat M, Le Dévéhat $C$. Analyse critique des explorations complémentaires vasculaires chez le diabétique. J Mal Vasc 2001;26:122-5.

35. Wu HD, Katz SD, Beniaminovitz A, Khan T, DiTullio MR, Homma S. Assessment of endothelium mediated vasodilatation of the peripheral circulation by transcutaneous ultrasonography and venous occlusion pletysmography. Heart Vessels 1999; 14:143-8.

36. Sandman DD, Shore AC, Tooke JE. Relation of skin capillary pressure in patients with insulin dependent diabetes mellitus to complications and metabolic control. N Eng J Med 1992;327:760-4.

37. Rubanyu GM. The role of endothelium in cardiovascular homeostasis and diseases. J Cardiovasc Pharmacol 1993:22:S1-14.

38. Tooke JE, Goh KL. Endoteliopathy precedes type 2 diabetes. Diabetes Care 1998;2:2047-9.

39. Tooke JE. Microcirculation and diabetes. Br Med Bull $1989 ; 45$.

40. Tooke JE, Hannemann MM. Adverse endothelial function and the insulin resistance syndrome. J Intern Med 2000;247:425-33.

\section{Endereço para correspondência:}

Vera Lucia Rabello de Castro Halfoun

Rua Professor Ortiz Monteiro 276, bloco C, apt.319

22245-100 Rio de Janeiro, RJ. 